\title{
A MORPHOMETRIC ANALYSIS OF THE GENICULATE BODIES IN SELECTED MAMMALIAN SPECIES
}

\author{
JANUSZ NAJDZION, BARBARA WASILEWSKA, KRYSTYNA BOGUS-NOWAKOWSKA, \\ MACIEJ RÓWNIAK, WITOLD ŻAKOWSKI, AND ANNA ROBAK \\ Department of Comparative Anatomy, \\ University of Warmia and Mazury, 10-727 Olsztyn, Poland, \\ jnajdzion@uwm.edu.pl
}

Received: December 5, 2011

Accepted: April 27, 2012

\begin{abstract}
Unbiased stereological methods were used to examine and compare morphometrically the geniculate bodies (GB) in representatives of four mammalian orders (Insectivora, Rodentia, Lagomorpha, and Carnivora). The significant disproportion was observed between the relative sizes of both geniculate nuclei and their neuronal populations in the common shrew and the bank vole. The medial geniculate body (MGB) in the common shrew definitely surpassed the lateral geniculate body (LGB) in terms of percentage volume and percentage number of neurons. The volume of the GB and their nuclei correlated with their mean neuronal populations, whereas the negative correlation was observed between volumes and neuronal density; however, not as distinct as in the non-sensory brain structures. In all examined species, the LGB always had a higher numerical density than the MGB, while the MGB neurons were always distinctly larger than that of the LGB, which clearly differentiated both neuronal complexes. Analysis of these data shows that the GB differs in terms of the morphometric characteristics in the studied species.
\end{abstract}

Key words: mammals, geniculate bodies, morphometric analysis.

The geniculate bodies (GB) of mammals form oval protrusions situated on the posterolateral surface of the thalamus. There are two geniculate bodies: the lateral geniculate body (LGB) and the medial geniculate body (MGB). Both of these neuronal structures consist of numerous subnuclei $(10,11,19,23)$, which are typically relay stations whose principal function is to process and regulate the flow of sensory information and relay it to the primary cortex. The LGB is mainly involved in visual processing $(9,13,16)$, but it also plays a major role in mediating photic and nonphotic phase shifts of circadian rhythms $(4,5,21)$. The MGB relays auditory information related to sound location, intensity, and frequency $(8,12,18)$.

In our previous papers $(14,15)$ we described in detail the topography and morphometric features of the LGB and MGB subnuclei. In the present study our goal was to characterise morphometrically the GB and their neurons as a whole integrated neural complex, which would summarise our work on these sensory structures. The integration of visual information with auditory information is crucial for most mammals to survive in their environment. Thus, knowledge about the correlation between the morphometric features of these sensory structures in different mammals would be very useful for better understanding the sensory systems. In the present study the GB of representatives of four phylogenetically different placental mammalian orders were morphometrically compared. Knowledge about the number of cells, cytoarchitecture and exact sizes and location of visual and auditory nuclei could support selective labelling methods such as immunocyto- and immunohistochemistry. Additionally, a validated morphometric model of these nuclei in the rabbit and fox could be very useful for studying the sensory systems and the pathogenesis of their degenerative diseases, since these animals are commercially bred and could serve as model organisms.

\section{Material and Methods}

The study was performed on 20 brains of the four species: the common shrew (Sorex araneus Insectivora), the bank vole (Clethrionomys glareolus Rodentia), the rabbit (Oryctolagus cuniculus Lagomorpha), the fox (Vulpes vulpes - Carnivora). Each species was represented by five adult animals of both sexes of the following body weight: the common shrew $11-12 \mathrm{~g}$, the bank vole $19-24 \mathrm{~g}$, the rabbit $3.5-4.5 \mathrm{~kg}$, the fox $8-10 \mathrm{~kg}$. The first two species were wild-living animals that were caught according to the permission of the Polish Environment Ministry. The remaining two species were animals obtained from commercial sources: the Vienna Blue rabbit and the silver fox. All efforts were made to minimise animal suffering and to use only the number of animals necessary to produce reliable scientific data.

Surgical procedures followed the guidelines established by the Animal Care and Use Ethical Committee of the University of Warmia and Mazury. All animals were given intraperitoneally a lethal dose $(80 \mathrm{mg} / \mathrm{kg}$ ) of Nembutal (Lundbeck, Denmark) and then 
they were decapitated. The brains were removed from skulls and immediately placed into $10 \%$ buffered formalin for immersion fixation for at least 2 months. In case of the rabbit and fox, the brains were cut into two sagittal halves before placing in the fixative. After the fixation, all brains were dehydrated in series of graded ethanols and embedded in paraffin. Next, they were coronally cut on a microtome (Leica, Germany) into serial $50 \mu \mathrm{m}$ sections and then stained with the cresyl violet. The Nissl staining was chosen as the best quantitative method for measuring cells, since it stains unspecifically all perikarya. All sections were analysed cytoarchitectonically (to define the boundaries of the GB) and morphometrically with a calibrated image analysis system that consisted of a computer equipped with morphometric software (Multi-Scan 8.2, Computer Scanning Systems, Poland) and a light microscope coupled with a digital camera (CM40P, VideoTronic, Germany). The brain of each animal was treated identically in accordance with the following standardised procedures:

Computer reconstructions of the lateral geniculate body. The partial microscopic images (the 512 × 512 pixel size) of a single brain section were digitally recorded by means of a camera attached to a microscope and a computer. All recorded images were joined into one large digital section that represented the whole GB and the adjoing structures. In order to obtain as exact as possible corresponding brain areas, every section in the series of histological preparations was digitally reconstructed and stored in a form of computer images.

Volumetric analysis. The total volume of the GB and its individual nuclei were calculated according to the formula proposed by DeVito et al. (3). The boundaries of the individual geniculate nuclei were outlined on examined digital sections by a mouse-driven cursor and their areas were calculated by computer with the morphometric software (Multi-Scan 8.2). To obtain the most accurate total volume, each digital section in the series was sampled.

Estimation of numerical density. The numerical density of cells in each of the LGN nuclei was estimated using the optical disector method described in details by West and Gundersen (22).

Estimation of the total number of neurons. The total number of neurons in each of the examined nuclei was calculated by multiplying estimates of the numerical density with the volume (22).

Morphometric parameters of the neurons. The morphometric parameters of the geniculate cells were measured and analysed using the calibrated system and image-analysing computer programme (Multi-Scan 8.2). The morphometric analysis was performed with the x40 (fox, rabbit) and x100 (common shrew, bank vole) objectives. The first section in the series was randomly selected and then every second (common shrew, bank vole) and fifth (rabbit, fox) serial section was investigated for a single animal. All neurons with distinct nuclei were selected for analysing. The identified neuronal cells were digitally outlined and measured. The analysed neuronal parameters were as follows: the length (the long axis of the soma), the width (the short axis of the soma), the area of soma, the circumference of soma, and the shape factor (the ratio of both axes).

The statistical analysis. Statistics were analysed with Statistica v.5.0 software (Statsoft, USA). All results were expressed as mean value \pm standard error of the mean (SEM). Statistical differences between groups of data were evaluated with ANOVA followed by post-hoc Duncan's tests and paired Student's $t$-tests. Correlation coefficients (r) between several morphometric parameters were estimated. The significance level was set at $\mathrm{P}<0.05$.

\section{Results}

Since the detailed morphometric parameters of the geniculate bodies and their cells are shown in Tables 1, 2 and Figs 1, 2, the results of the remaining data may be summarised as follows:

Volume. The volumes and percentages of volumes of the LGB and MGB were significantly different $(\mathrm{P}<0.05)$ among examined species, except for the fox that did not differ significantly (volume: $\mathrm{P}>0.35$, percentage of volume: $\mathrm{P}>0.14$ ).

Numerical density. The mean numerical densities of the LGB and MGB were significantly different $(\mathrm{P}<0.05)$ among examined species. The mean neuronal densities of the LGB in the common shrew, rabbit, and fox were significantly higher $(\mathrm{P}<0.05)$ than the mean neuronal density of the GB in these species, while in the bank vole this parameter was not significantly different $(\mathrm{P}>0.06)$. As for the MGB, these parameters in common shrew, rabbit, and fox were significantly lower $(\mathrm{P}<0.05)$, while in the bank vole it did not differ significantly $(\mathrm{P}>0.06)$. The neuronal density of the GB was negatively correlated with its volume $(\mathrm{r}=-0.86 ; \mathrm{P}<0.14)$.

Total number of neurons. In all species the mean percentage contributions of the LGB and MGB to the total neuronal populations of the GB were significantly different $(\mathrm{P}<0.05)$. The mean cell population of the GB was positively correlated with its mean volume $(\mathrm{r}=0.99 ; \mathrm{P}<0.005)$. There was a correlation between the growth of the LGB volume and increase in the mean neuronal population of the LGB ( $\mathrm{r}=0.99$; $\mathrm{P}<0.003$ ). The similar correlation was observed in case of the MGB $(\mathrm{r}=0.99 ; \mathrm{P}<0.007)$.

Morphometric parameters of geniculate neurons. All morphometric parameters of the LGB cells in the studied species were significantly lower $(\mathrm{P}<0.05)$ than the respective means of the GB in this species, while in the case of the MGB they were higher $(\mathrm{P}<0.05)$. The correlation was observed between the growth of the LGB volume and increase in the LGB neuronal sizes (area of soma: $\mathrm{r}=0.88 ; \mathrm{P}<0.12$; circumference of soma: $\mathrm{r}=0.90 ; \mathrm{P}<0.10$; length: $\mathrm{r}=0.94 ; \mathrm{P}<0.06$; width: $\mathrm{r}=0.81$; $\mathrm{P}<0.19$ ). The sizes of the MGB neurons grew correlatively with an increase in the MGB volume as well $(\mathrm{r}=0.90 ; \mathrm{P}<0.09 ; \mathrm{r}=0.89 ; \mathrm{P}<0.11 ; \mathrm{r}=0.87 ; \mathrm{P}<0.13$; $\mathrm{r}=0.93 ; \mathrm{P}<0.07$, respectively). 
Table 1

Morphometric parameters of the GB

\begin{tabular}{ccccc}
\hline & Common shrew & Bank vole & Rabbit & Fox \\
\hline Volume of GB $\left(\mathrm{mm}^{3}\right)$ & $0.21 \pm 0.01$ & $0.58 \pm 0.02$ & $19.43 \pm 0.4$ & $42.44 \pm 0.7$ \\
\hline Volume of LGB $\left(\mathrm{mm}^{3}\right)$ & $0.05 \pm 0.003$ & $0.22 \pm 0.01$ & $9.03 \pm 0.2$ & $21.42 \pm 0.4$ \\
\hline Volume of MGB $\left(\mathrm{mm}^{3}\right)$ & $0.16 \pm 0,01$ & $0.36 \pm 0.006$ & $10.40 \pm 0.2$ & $21.02 \pm 0.3$ \\
\hline Density of GB $\left(\mathrm{N} / \mathrm{mm}^{3}\right)$ & $224,036 \pm 4,224$ & $176,157 \pm 5,092$ & $23,442 \pm 785$ & $26,013 \pm 1,151$ \\
\hline Density of LGB $\left(\mathrm{N} / \mathrm{mm}^{3}\right)$ & $262,158 \pm 4,934$ & $186,326 \pm 6,942$ & $28,111 \pm 756$ & $32,493 \pm 1,188$ \\
\hline Density of MGB $\left(\mathrm{N} / \mathrm{mm}^{3}\right)$ & $185,914 \pm 3,515$ & $165,989 \pm 3,241$ & $18,773 \pm 814$ & $19,534 \pm 1,114$ \\
\hline Number of GB neurons (N) & $42,322 \pm 1,736$ & $98,953 \pm 2,092$ & $443,083 \pm 9,837$ & $1,101,963 \pm 28,978$ \\
\hline Number of LGB neurons (N) & $12,028 \pm 366$ & $38,779 \pm 1,377$ & $248,188 \pm 4,302$ & $668,941 \pm 11,074$ \\
\hline Number of MGB neurons (N) & $30,293 \pm 1,370$ & $60,174 \pm 714$ & $194,895 \pm 5,534$ & $433,022 \pm 17,904$ \\
\hline
\end{tabular}

Table 2

Morphometric parameters of the GB neurons

\begin{tabular}{|c|c|c|c|c|}
\hline & Common shrew & Bank vole & Rabbit & Fox \\
\hline GB - area of soma $\left(\mu \mathrm{m}^{2}\right)$ & $59 \pm 6$ & $78 \pm 8$ & $180 \pm 16$ & $187 \pm 23$ \\
\hline LGB - area of soma $\left(\mu \mathrm{m}^{2}\right)$ & $57 \pm 6$ & $73 \pm 6$ & $151 \pm 7$ & $157 \pm 16$ \\
\hline MGB - area of soma $\left(\mu \mathrm{m}^{2}\right)$ & $62 \pm 5$ & $90 \pm 3$ & $199 \pm 12$ & $208 \pm 21$ \\
\hline GB - circumference of soma $(\mu \mathrm{m})$ & $31 \pm 2$ & $34 \pm 2$ & $55 \pm 3$ & $56 \pm 3$ \\
\hline LGB - circumference of soma $(\mu \mathrm{m})$ & $30 \pm 2$ & $33 \pm 1$ & $49 \pm 1$ & $52 \pm 1$ \\
\hline MGB - circumference of soma $(\mu \mathrm{m})$ & $32 \pm 1$ & $37 \pm 0.5$ & $58 \pm 2$ & $58 \pm 2$ \\
\hline GB - length of soma $(\mu \mathrm{m})$ & $11 \pm 0.7$ & $12 \pm 0.7$ & $20 \pm 1$ & $20 \pm 1$ \\
\hline LGB - length of soma $(\mu \mathrm{m})$ & $11 \pm 0.5$ & $12 \pm 0.5$ & $18 \pm 0.3$ & $20 \pm 0.3$ \\
\hline MGB - length of soma $(\mu \mathrm{m})$ & $12 \pm 0.3$ & $13 \pm 0.2$ & $22 \pm 1$ & $21 \pm 1$ \\
\hline GB - width of soma $(\mu \mathrm{m})$ & $7 \pm 0.5$ & $9 \pm 0.4$ & $13 \pm 0.5$ & $13 \pm 1$ \\
\hline LGB - width of soma $(\mu \mathrm{m})$ & $7 \pm 0.4$ & $8 \pm 0.4$ & $12 \pm 0.4$ & $11 \pm 0.9$ \\
\hline MGB - width of soma $(\mu \mathrm{m})$ & $7 \pm 0.4$ & $9 \pm 0.2$ & $13 \pm 0.3$ & $14 \pm 0.7$ \\
\hline GB - shape factor & $1.51 \pm 0.05$ & $1.45 \pm 0.03$ & $1.61 \pm 0.05$ & $1.65 \pm 0.12$ \\
\hline LGB - shape factor & $1.46 \pm 0.02$ & $1.44 \pm 0.04$ & $1.51 \pm 0.03$ & $1.82 \pm 0.14$ \\
\hline MGB - shape factor & $1.60 \pm 0.04$ & $1.47 \pm 0.02$ & $1.68 \pm 0.04$ & $1.54 \pm 0.02$ \\
\hline
\end{tabular}




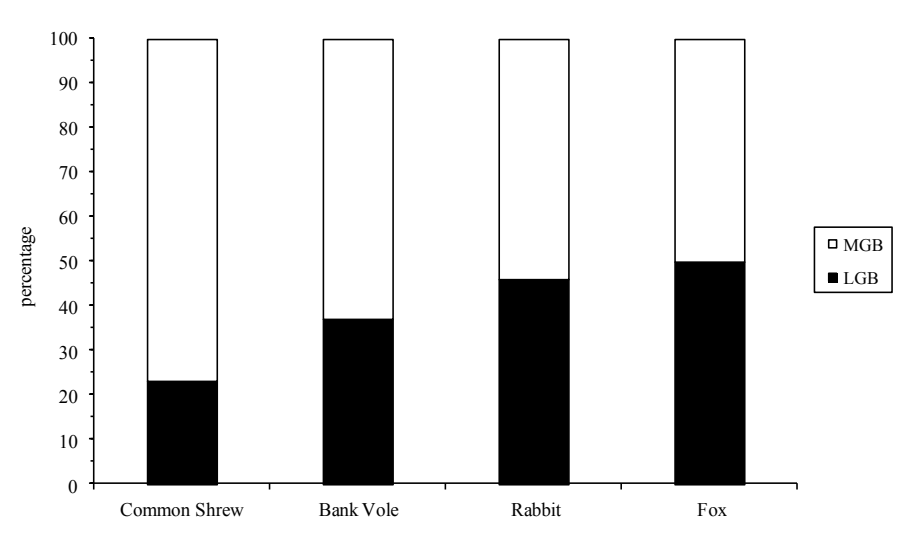

Fig. 1. Percentages of the LGB and MGB volumes in the total GB volume.

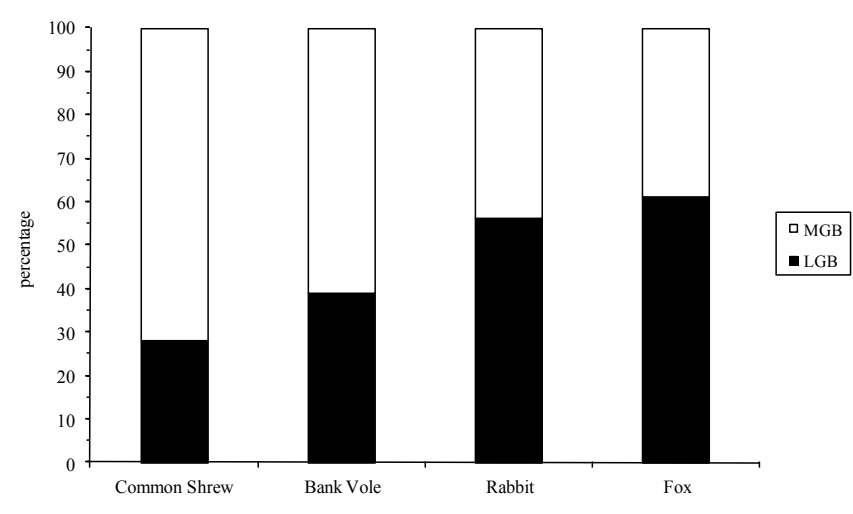

Fig. 2. Percentages of the LGB and MGB neurons in the total number of GB neurons.

\section{Discussion}

The LGB is not a homogeneous structure, since it is separated into two main divisions: the dorsal nucleus (LGBd) and the ventral nucleus (LGBv). The comparative anatomy studies have revealed that the highly organised visual system of carnivores has many features in common with that of primates. All species from these two orders have multilayered structure of the LGBd and also most of them have eyes positioned on the front of their heads, which enables binocular vision $(2,11,13)$. In the studied species, the LGBd of the fox had the most complicated morphology and multilayered structure, which is typical for carnivores (14). The LGBd of most mammals that have been examined so far is not arranged in any laminated pattern, which is believed to be a primitive, ancestral condition (1). In the common shrew and the bank vole, the LGBd had a homogeneous structure and no cytoarchitectonic lamination could be observed (14). Similar structure of the LGBd was observed in the rabbit; however, it was noticeably larger and it could be subdivided into two parts in the middle section, whereas the rostral and caudal poles of the LGBd were cytoarchitecturally uniform (14). Thus, from the anatomical point of view, the rabbit is located between mammals with homogeneous and multilayered structure of the LGBd. From the functional point of view, mammals with the multilayered LGBd have a highly developed visual system and a proportionally big part of ipsilaterally projecting retinogeniculate fibres, while mammals with the non-layered LGBd are less visually oriented (1). The MGB relays auditory information between the inferior colliculus and the auditory cortex. It is a heterogenous structure that is made up of three main divisions: the dorsal nucleus (MGBd), the ventral nucleus (MGBv), and the medial nucleus (MGBm). Despite similar nomenclature, there is some discrepancy in terms of determination and classification of the boundaries of the MGB nuclei in different mammals. The main reason for this divergence seems to be related to the phylogenetic development and differentiation of the thalamus. However, the results reported in the literature confirm that at least the basic, tripartite pattern of structural organisation of the MGB is similar in all mammals (8, 19, 23). Additionally, these three main divisions are comparable in different species, on the basis of their cytoarchitecture, neuronal architecture, connectivity, and neurochemistry (23). Despite this similarity, there are some significant differences in the morphometric parameters of the MGB nuclei, as well as in the case of the LGB nuclei $(14,15)$.

In all examined species, the mean volume of the GB ranged between 0.2 and $42 \mathrm{~mm}^{3}$ and it grew with an increase in the total brain volume. The smallest GB volume was observed in the common shrew, while the largest in the fox. The contribution of the LGB to the total volume of the GB ranged from the lowest of $23 \%$ in the common shrew to the largest of $51 \%$ in the fox. The relative sizes of the MGB were also different in each species. In comparison with the LGB, the percentage contribution of the MGB showed a reverse tendency with the lowest value in the fox $(49 \%)$, and the predominance in the common shrew (77\%). In the rabbit and the fox, the percentage contribution of the LGB and the MGB to the total volume of the GB was very similar (about 50\%), whereas in the remaining two species, it was considerably different, reaching its highest disproportion in the common shrew. In the presented study, the neuronal density of the GB was negatively correlated with the volumes of the GB as well as brain sizes. The exception was fox, which despite having the larger volumes of geniculate nuclei than rabbit, had the higher number of neurons per $\mathrm{mm}^{3}$. In all examined species, the LGB always had a higher numerical density than the MGB that clearly differentiated both neuronal complexes. A decrease in neuronal density corresponding to a simultaneous increase in volume has been described by other authors in several different mammalian brain regions $(6,17,20)$. However, as our previous study $(14,15)$ and this paper have shown, the negative correlation between the volume and numerical density in the sensory centres of the brain might not be as distinct as in the non-sensory brain structures. In the present study, the volume of the GB and their nuclei correlated with their mean neuronal populations. Thus, the smallest number of the GB neurons was observed in 
the common shrew, while the largest was found in the fox. This corresponds to other studies, where similar correlations were observed in different brain areas. The percentage contribution of the LGB and MGB cells to the total neuronal population of the GB was different in each species. In the rabbit and the fox, there was a larger contribution from the LGB, whereas in the common shrew and the bank vole, the MGB was predominant. The available literature with morphometric data from the GB is very scarce, dealing only with the neuronal population of rat MGB nuclei (7). The total number of neurons in the MGB of this species was estimated as 72.300 , which is the most similar to the bank vole in the presented study. The mean size of the GB neurons, that was the resultant of the length, width, area, and circumference of soma, grew correlatively to the volumes of the GB in the studied mammals. Thus, the smallest neurons were found in the common shrew, while the largest were observed in the fox. Furthermore, the MGB neurons were always distinctly larger than that of the LGB. The somas of GB neurons in the common shrew and the bank vole had similar shapes. The analogous similarity of the neuronal somas was observed between the rabbit and the fox. However, the later species had a noticeable higher shape factor, meaning that elongated neurons are more numerous in the rabbit and fox.

The visual and auditory systems frequently work together to facilitate the identification and localisation of objects and events in the external world. As was shown in this study, there are some significant differences in the morphometric features such as percentages of volumes, numerical densities, and proportions of neuronal populations of the GB nuclei in the studied species. It is noteworthy that there is a striking difference in the percentage contribution of the LGB and MGB volumes and cells between the common shrew and the fox. The present results seem to indicate that this type of discrepancy could mainly exist between carnivores and insectivores, which might be especially interesting from a phylogenetic perspective. However, we should not forget that the GB are typical sensory centres. Therefore, their relative sizes and proportions might be to a large extent related to specific environmental conditions in which animals live. Insectivores are the most ancestral mammals, which are oriented mainly olfactorically, while carnivores are predators, which have a highly specialised visual system and heavily rely on visual information. The representatives of rodents and lagomorphs are located between the representatives of insectivores and carnivores in terms of morphometric parameters.

\section{References}

1. Brauer K., Schober W., Winkelmann E.: Phylogenetical changes and functional specializations in the dorsal lateral geniculate nucleus (dLGN) of mammals. J Hirnforsch 1978, 19, 177-187.

2. Conley M., Birecree E., Casagrande V.A.: Neuronal classes and their relation to functional and laminar organization of the lateral geniculate nucleus: a Golgi study of the prosimian primate, Galago crassicaudatus. J Comp Neurol 1985, 242, 561-583.

3. DeVito J.L., Graham J., Sackett G.P.: Volumetric growth of the major brain divisions in fetal Macaca nemestrina. J Hirnforsh 1989, 30, 479-487.

4. Edelstein K., Amir S.: The role of the intergeniculate leaflet in entrainment of circadian rhythms to a skeleton photoperiod. J Neurosci 1999, 19, 372-380.

5. Goel N., Governale M.M., Jechura T.J., Lee T.M.: Effects of intergeniculate leaflet lesions on circadian rhythms in Octodon degus. Brain Res 2000, 877, 306313.

6. Kowiański P., Dziewiątkowski J., Kowiańska J., Moryś J.: Comparative anatomy of the claustrum in selected species: A morphometric analysis. Brain Behav Evol 1999, 53, 44-54.

7. Kulesza R.J., Viñuela A., Saldaña E., Berrebi A.S.: Unbiased stereological estimates of neuron number in subcortical auditory nuclei of the rat. Hear Res 2002, 168, 12-24.

8. LeDoux J.E., Ruggiero D.A., Reis D.J.: Projections to the subcortical forebrain from anatomically defined regions of the medial geniculate body in the rat. J Comp Neurol 1985, 242, 182-213.

9. Livingston C.A., Fedder S.R.: Visual-ocular motor activity in the macaque pregeniculate complex. J Neurophysiol 2003, 90, 226-244.

10. Livingston C.A., Mustari M.J.: The anatomical organization of the macaque pregeniculate complex. Brain Res 2000, 876, 166-179.

11. McConnell S.K., Le Vay S.: Anatomical organization of the visual system of the mink, Mustela vison. J Comp Neurol 1986, 250, 109-132.

12. Mello L.E., Tan A.M., Finch D.M.: Convergence of projections from the rat hippocampal formation, medial geniculate and basal forebrain onto single amygdaloid neurons: an in vivo extra- and intracellular electrophysiological study. Brain Res 1992, 587, 24-40.

13. Murphy P.C., Duckett S.G., Sillito A.M.: Comparison of the laminar distribution of input from areas 17 and 18 of the visual cortex to the lateral geniculate nucleus of the cat. J Neurosci 2000, 20, 845-853.

14. Najdzion J., Wasilewska B., Bogus-Nowakowska K., Równiak M., Szteyn S., Robak A.: A morphometric comparative study of the lateral geniculate body in selected placental mammals: the common shrew, the bank vole, the rabbit, and the fox. Folia Morphol (Warszawa) 2009, 68, 70-78.

15. Najdzion J., Wasilewska B., Równiak M., BogusNowakowska K., Szteyn S., Robak A.: A morphometric comparative study of the medial geniculate body of the rabbit and the fox. Anat Histol Embryol 2011, 40, 326334.

16. Nakamura H., Itoh K.: Cytoarchitectonic and connectional organization of the ventral lateral geniculate nucleus in the cat. J Comp Neurol 2004, 473, 439-462.

17. Narkiewicz O., Dziewíątkowski J., Moryś J.: Lateral tuberal nucleus in man and macaca comparative morphometric investigations. Folia Morphol (Warszawa) 1994, 53, 1-12.

18. Niimi K., Ono K., Kusunose M.: Projections of the medial geniculate nucleus to layer 1 of the auditory cortex in the cat traced with horseradish peroxidase. Neurosci Lett 1984, 45, 223-228.

19. Radtke-Schuller S.: Cytoarchitecture of the medial geniculate body and thalamic projections to the auditory 
cortex in the rufous horseshoe bat (Rhinolophus rouxi). Anat Embryol (Berlin) 2004, 209, 59-76.

20. Równiak M., Robak A., Szteyn S., Bogus-Nowakowska K., Wasilewska B., Najdzion J.: The morphometric study of the amygdala in the rabbit. Folia Morphol (Warszawa) 2007, 66, 44-53.

21. Vrang N., Mrosovsky N., Mikkelsen J.D.: Afferent projections to the hamster intergeniculate leaflet demonstrated by retrograde and anterograde tracing. Brain Res Bull 2003, 59, 267-288.

22. West M.J., Gundersen H.J.: Unbiased stereological estimation of the number of neurons in the human hippocampus. J Comp Neurol 1990, 296, 1-22.

23. Winer J.A., Kelly J.B., Larue D.T.: Neural architecture of the rat medial geniculate body. Hear Res 1999, 130, $19-41$. 